\title{
Ant Colony Optimization Algorithm for Robot Path Planning
}

\author{
Q.L. Xu, D.X. Zhang \\ College of Automation and Electronic Engineering \\ Qingdao University of Science and Technology \\ Qingdao, China
}

\begin{abstract}
In this article two different optimization algorithms are presented to solve the deficiency of ant colony algorithm such as slow convergence rate and easy to fall into local optimum. This method based on Max-Min Ant System, established an adaptive model for pheromone evaporation coefficient adjusted adaptively and avoided the ants falling into local optimum. At the same time, this optimization algorithm used the strategy of the survival of the fittest way to optimize the pheromone update mechanism to accelerate the convergence rate. Finally, by comparison with ant colony algorithm, the simulation results show that, both the optimal path and routing time are optimized, and proved that the optimization algorithm is valid and feasible.
\end{abstract}

Keywords- path planning; ant colony optimization algorithm; robot; Max-Min Ant System

\section{INTRODUCTION}

Path planning is the concept of searching the optimal path from a given starting point to the ending point without collision in an obstacle environment [1]. At present, many domestic and foreign scholars have developed several algorithms to solve the path planning problem, such as the artificial potential field method, genetic algorithm, fuzzy logic algorithm, neural network algorithm and ant colony algorithm [2], but all of them have pros and cons.

Ant colony algorithm was presented by the Italian scholar Dorigo in his doctoral thesis in 1991 [3], which was inspired by the behaviour of the ants finding the path in the process of looking for food. Aiming at solving the shortcomings of ant colony algorithm, many scholars have put forward the improvement methods. Li Guo have introduced the combination of the deterministic selection with random selection not only solved the ants falling into local optimum [4], but also increased the diversity of solution, but it cannot guarantee the integrity of the algorithm. Zhang Meiyu proposed a combination of ant colony algorithm and MMAS [5], this optimization algorithm averaged the probability of each path which will be selected, but it was not conducive to the rapid convergence of the algorithm.
This paper analyses the existing problems of ant colony algorithm. At first, this method optimizes the pheromone update mechanism to avoid the single mechanical equal distribution of pheromone which may reduce the convergence rate of ant colony algorithm, and then, it uses the Max-Min Ant System to solve the ants trapped into local optimum, so the ants can get a better choice between the maintain of the former path with looking for a new path based on the actual situation. Finally, simulation experiments are carried out to verify this optimization algorithm.

\section{ENVIRONMENT DESCRIPTION}

Robot path planning including environment modeling and path search in the environment model. The main methods of environment modeling include visibility graph method, the free space method and the grid method [6]. This paper uses the grid method, because the grid method has the advantages of high precision, easy to implement compared with the previous two modeling methods. The workspace in this paper is a limited area of $20 \times 20$ square grid on the $2 \mathrm{D}$ region with may contain obstacles, as shown in Figure 1:

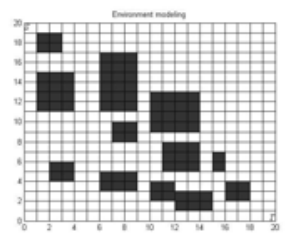

FIGURE I. ENVIRONMENT MODELLING

\section{Ant Colony Optimization Algorithm}

This optimization method uses the combination of Pheromone update optimization mechanism and MMAS, which greatly improved the performance of ant colony algorithm. The specific optimization methods are described below. 


\section{A. Using Adaptive Adjustment to Enhance the Vitality of the Algorithm}

Ant colony algorithm is a positive feedback system, if the amount of pheromone on the optimal path was enhanced continuously will lead to stagnation, finding the better path will be pretty hard. Because when the pheromone evaporation coefficient $\rho$ is too large, the ants tend to choose the path which had been searched previously, this will affect the algorithm's global search ability. While reducing $\rho$ can improve the algorithm's global search ability, but it will reduce the convergence rate of the algorithm. To solve this problem, the idea of MMAS was used to adjust the $\rho$ according to actual situation. At first, the amount of pheromone on each path will limit within the range of $\left[\tau_{\min }, \tau_{\max }\right]$, and then, the pheromone evaporation coefficient was adjusted adaptively. The initial value of $\rho$ is $\rho\left(t_{0}\right)=0.95$, when the optimal path minPL has not changed in $\mathrm{N}$ cycles, using the following formula to adjust:

$$
\rho(t)=\left\{\begin{array}{l}
0.95 \rho(t-1) \text {, when } 0.95 \rho(t-1) \geq 0.4 \\
0.4, \text { otherwise }
\end{array}\right.
$$

While, the minimum value for $\rho$ is 0.4 , the minimum value was used to prevent the decrease of convergence rate when $\rho$ is too small. This method can solve the ant colony trapped into local optimum.

\section{B. Using Survival of the Fittest Strategy to Accelerate the Convergence Rate}

The ants will not falling into local optimum after the adaptive adjustment, but the convergence rate will be reduced if $\rho$ was still decreased continuously. To compensate these deficiencies of the above optimization method, the attenuation coefficient $\theta$ is proposed. It was used to adjust the amount of pheromone on each path, we strengthen the optimal path pheromone quantity and decrease the poor path pheromone quantity moderately at the end of each cycle, and the value of $\theta$ is generally between 0 and 1 . After the end of each cycle, taking the average meanPL of all the paths, we define the paths are better paths which less than meanPL and the poor paths are greater than meanPL, using the following formula to update pheromone:

$$
\begin{gathered}
\Delta \tau_{i j}^{\prime}=\left\{\begin{array}{l}
\sum_{k=1}^{m} \Delta_{i j}^{k}[\theta, \text { ant } k \text { through poor path } \\
\sum_{k=1}^{m} \Delta_{i j}^{k}(1 / \theta), \text { ant } k \text { through better path }
\end{array}\right. \\
\tau_{i j}(t+n)=(1-\rho) \tau_{i j}(t)+\Delta \tau_{i j}^{\prime}
\end{gathered}
$$

We using this method to update pheromone, it can not only accelerate the convergence rate, but also can increases the probability of the better paths which will be selected, and most important, it won't completely rule out the possibility of other paths which may become the optimal path. The idea is that the ants will not trapped into the local optimum after the adjustment of $\rho$ and the convergence rate will be accelerated until the introduce of $\theta$. After the combination of these two optimization methods, this optimization algorithm accelerates the convergence rate of the algorithm under the premise, but also guarantees the integrity of the algorithm.

\section{Robot Path Planning Based on Ant Colony OPTIMIZATION ALGORITHM}

In robot path planning process, when the optimal path has not changed within a certain number of cycles, we using the formula (1) to adjust pheromone evaporation coefficient adaptively, and at the end of each cycle, using the equation (2) and (3) to update pheromone. After the optimization of the above rules, the next step is to combine the ant colony algorithm, specific steps are as follows:

(1) Initialization parameters: Initialize the parameters, and limit the amount of pheromone on each path within a range of $\left[\tau_{\min }, \tau_{\max }\right]$.

(2) Start cycle: Each ant moves according to the formula to determine which grid will be reach in the next step.

(3) The end of the cycle, average and comparison: When all the ants have completed the routing, calculate the average of all the paths, and compare each path with the average.

(4) Global pheromone update: After the comparison and classification, using the formula (2) and (3) to update the pheromone on each path.

(5) Judge and adjust: Judge whether the optimal path has been optimized in $\mathrm{N}$ cycles. And adjust the $\rho$ according to formula (1).

(6) Judge: Judge whether the cycle number reaches Ncmax. If achieved, output the optimum path, if not, returning to step (2) continue the iteration.



\section{FIGURE II. ANT COLONY OPTIMIZATION ALGORITHM FLOWCHART.}

\section{Simulation Results AND ANALysis}

According to the above optimization ideas, in order to verify the effectiveness of the optimization algorithm, we using Matlab make simulation compared with ant colony algorithm in a $2.53 \mathrm{GHz} \mathrm{CPU}$ and 4.00GB RAM computer. The experiment parameter are $\alpha=1, \beta=5$, the ant number $m=50, \rho=0.6$ (The initial pheromone of ant colony optimization algorithm $\rho\left(t_{0}\right)=0.95$ ), the total amount of information $Q=1$, the maximum number of cycles $N c \max =500$. The simulation results are shown below: 


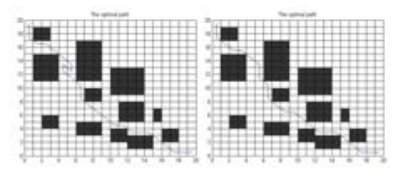

(a) The comparison of the optimal path.

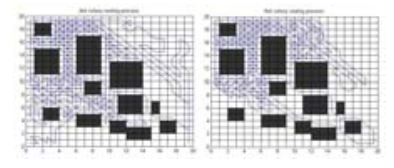

(b) The comparison of the routing process.



(c) The comparison of the convergence curve.

\section{FIGURE III. THE SIMULATION RESULTS COMPARISON.}

By comparing the simulation results, the optimal paths and routing time statistics in the table below:

TABLEI. THE SIMULATION RESUlTS COMPARISON.

\begin{tabular}{|c|c|c|}
\hline comparison & $\begin{array}{c}\text { optimal } \\
\text { path length }\end{array}$ & $\begin{array}{c}\text { routing } \\
\text { time }\end{array}$ \\
\hline $\begin{array}{c}\text { optimization } \\
\text { algorithm }\end{array}$ & 30.382 & $53.05 \mathrm{~s}$ \\
\hline $\begin{array}{c}\text { ant colony } \\
\text { algorithm }\end{array}$ & 32.624 & $68.05 \mathrm{~s}$. \\
\hline
\end{tabular}

According to the experimental simulation and statistical results, the optimal path is superior to the ant colony algorithm. By comparing the convergence rate, the optimization algorithm has been converged about 120 cycles, but the ant colony algorithm needs 170 cycles, it indicates that ants can find the optimal path faster. While comparing the ant routing process, the ant colony optimization algorithm avoids the blindness of the ant colony algorithm, it means that this optimization algorithm will reduce the probability of ants which may caught into a trap, proved that the optimization algorithm is superior to ant colony algorithm.

\section{CONCLUSION}

In this paper, ant colony optimization algorithm has been proposed to resolve the problems such as slow convergence speed, easy to fall into traps and local optimum. First, according to MMAS, the amount of pheromone on each path would limit into a certain range. At the same time, during the cycle, we judge whether the optimal path has been changed in a certain cycle times to decide whether to adjust the pheromone evaporation coefficient, this will avoid the ants trapped into local optimum and stagnation effectively. Then, after the end of each cycle, the amount of pheromone on each path has been adjusted reasonably, thus can not only ensure the integrity of the algorithm, but also can accelerate the convergence rate of the algorithm. Finally, the simulation experiment is carried out, the results show that the optimization algorithm is superior to ant colony algorithm in path length and routing time. So the optimization algorithm can be applied to the robot path planning effectively.

\section{ACKNOWLEDGEMENTS}

This work is supported by Scientific Research Foundation of Qingdao University of Science and Technology.

\section{REFERENCES}

[1] R. Wen, D.W. Li. Path Planning of Robot Based on Ant Colony Algorithm [J]. Computer \& Digital Engineering, pp. 20-22, 2012.

[2] S.Y. Li. Progresses in Ant Colony Optimization Algorithm with Applications [J]. Computer Measurement \& Control, pp. 911-913, 2003.

[3] H.B. Duan, D.B. Wang and X.F. Yu. Review on research progress in ant colony algorithm [J]. History of Natural Science, pp. 102-105, 2012.

[4] G. Li, S.J. Liu. Path Planning of Mobile Robot Based on Improved Ant Colony Algorithm [J]. Control Engineering of China, pp. 473-455, 485, 2005.

[5] M.Y. Zhang, H. Huang and Z.F. Hao. Motion Planning of Autonomous Mobile Robot Based on Ant Colony Algorithm [J]. Computer Engineering and Applications, pp. 34-37, 2005.

[6] C.M. Ren and J.X. Zhang. Robot Path Planning Based on Improved Ant Colony Optimization [J]. Computer Engineering, pp. 1-3, 35, 2008. 